\title{
Time-reversal Waveform Preconditioning for Clutter Rejection
}

\author{
T. Varslot, ${ }^{a}$ B. Yazıcı, ${ }^{a}$ C.-E Yarman, ${ }^{a}$ M. Cheney ${ }^{a}$ and L. Scharf. ${ }^{b}$ \\ ${ }^{a}$ Rensselaer Polytechnic Institute, Troy, NY, USA \\ ${ }^{b}$ Colorado State University, Fort Collins, CO, USA
}

\begin{abstract}
A time-reversal implementation of a transmit waveform preconditioning scheme for optimal clutter rejection in radar imaging is presented. Waveform preconditioning involves determining a map on the space of transmit waveforms, and then applying this map to the waveforms before transmission. Our work applies to antenna arrays with an arbitrary number of transmit- and receive elements, and makes no assumptions about the elements being co-located. Waveform preconditioning for clutter rejection achieves efficient use of power and computational resources by distributing power properly over a frequency band and by eliminating clutter filtering in receive processing. By our time-reversal implementation we avoid the need to obtain an explicit model for the environment in order to compute the preconditioning operator.
\end{abstract}

\section{INTRODUCTION}

In earlier work we introduced waveform preconditioning of transmit waveforms for optimal clutter rejection [1], [2]. Here we show how preconditioning of transmit waveforms for optimal clutter rejection can be performed using a time-reversal approach. Waveform preconditioning involves determining a map on the space of transmit waveforms, and then applying this map to the waveforms before transmission. Explicit expressions for the preconditioning operator involve the Green's function for the background medium, as well as second-order statistics for the target of interest and the clutter. Here we show that the need for such explicit information can be avoided by a time-reversal approach to computing the preconditioning operator.

In radar applications the scene (everything in the radar beam) is decomposed of three classes: objects of interest, objects which are not of interest, and (known) background. Objects of interest are referred to as targets, while those objects which are not of interest are referred to as clutter. In this paper, scattering from clutter is an undesired feature to be rejected by means of a waveform preconditioning operator. Clutter rejection is an important task, as scattering from clutter can overpower scattering from targets, thus rendering the targets difficult to detect or image. Our primary application is radar imaging. It should be clear, however, that our physics-based approach is applicable to pulse-echo imaging in general, e.g. ultrasound imaging, sonar imaging and microwave imaging.

The time-reversal principle is based on an invariance to changing the sign of the temporal variable in the wave equation [3], [4]. It has been used in many applications which involve wavepropagation, e.g., ultrasound imaging [4], [5], [6], [7], underwater acoustics [8], [9], radar imaging [10], [11], and microwave imaging [12]. Typically, time-reversal applications work well in a multiple-scattering medium and where explicit modeling of the medium is difficult due to its complexity or due to random perturbations [7], [13], [14], [15].

We formulate the processing of radar data from an arbitrary number of transmit- and receive-antennas in a stochastic framework. In this sense our work is closely related to Yazici et al. [2], where an a priori stochastic characterization of the scattering distribution was used to construct optimal waveforms for mono-static rangeDoppler imaging. We then show how we by re-transmitting our scattering measurements can exploit the time-reversal principle to determine the preconditioning operator without explicitly knowing the envoronment.

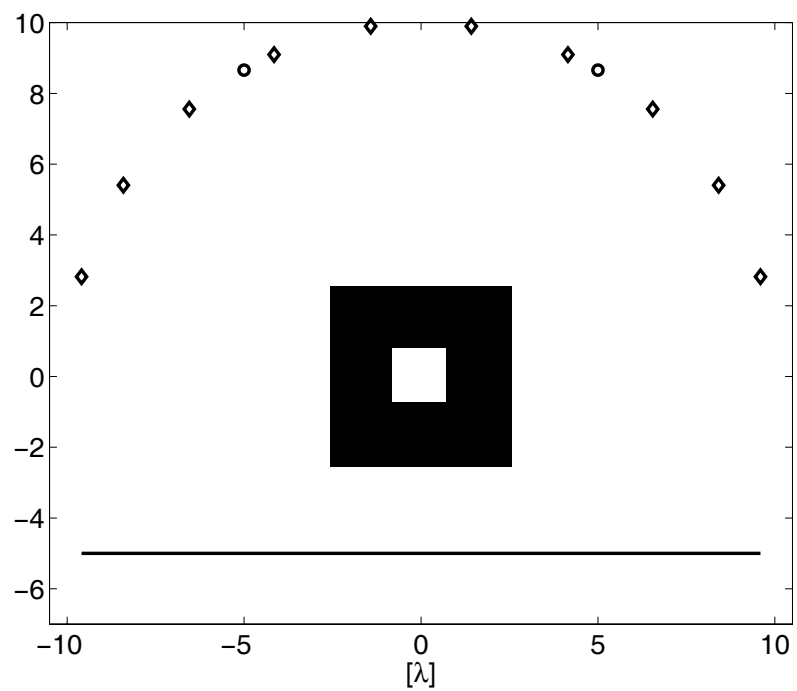

Fig. 1. An antenna array with two transmitting elements (circles) and ten receiving elements (diamonds). The elements are placed at equidistant points along a an arc with radius $10 \lambda$. The target is indicated as a square with sides of $1.5 \lambda$, while the region of interest is $5 \lambda \times 5 \lambda$ around the target. This region is filled with clutter. The solid straight line indicates the location of a reflective mirror in our simple multipath scenario.

From a communications point of view, the radar transmit signal which illuminates the target may be considered as a means for establishing a communications channel between the target and the observer. In this language, the effect of a complex environment is considered as part of this communications channel. The goal is to design a filter which, when applied to the transmit signal, virtually shapes the received signal in a desired manner. Thus, there are obvious similarities between the ideas presented here for radar imaging, and the existing literature on precoding.[16], [17], [18]

\section{PRELIMINARY NOTATION AND MODELING}

\section{A. Antenna array}

We consider an antenna array consisting of $m$ transmitting elements and $n$ receiving elements. The placement of these elements can be chosen arbitrarily, i.e., the location of each element may be assigned independently of where the other elements are located. For expository convenience we assume each element to behave like an isotropic point antenna, i.e., radiation patterns from each element do not exhibit any directivity.

In order to exploit the spatial diversity inherent in the antenna, it is desirable to allow for transmitting different waveforms from each element. Let $s_{j}(t)$ denote the waveform which emanates from the $j^{\text {th }}$ element. We arrange the transmit waveforms in a transmit vector $\boldsymbol{s}(t)$

$$
\boldsymbol{s}(t)=\left[s_{1}(t), \ldots, s_{m}(t)\right]^{\mathrm{T}} .
$$


Similarly, if the measured scattering at the $i^{\text {th }}$ receive element is denoted by $m_{i}(t)$, then the scattering which is collected by the array may be arranged in a measurement vector $\boldsymbol{m}(t)$

$$
\boldsymbol{m}(t)=\left[m_{1}(t), \ldots, m_{n}(t)\right]^{\mathrm{T}} .
$$

\section{B. Scattering model}

The ability to distinguish targets depends on how much their electromagnetic properties deviate from the background. We denote this deviation by the reflectivity function $V(\boldsymbol{x})$. At an abstract level, we denote by $\mathcal{H}(V)$ the operator which maps the transmit vector $\boldsymbol{s}(t)$ to the measurement vector $\boldsymbol{m}(t)$

$$
\boldsymbol{m}(t)=\mathcal{H}(V) \boldsymbol{s}(t) .
$$

An explicit relationship between the reflectivity function $V$ and the operator $\mathcal{H}(V)$ can be derived in terms of the Green's function $g(\boldsymbol{x}, \boldsymbol{y}, t)$ for the background medium. The Green's function is the response measured at position $\boldsymbol{x}$ from an impulse $\delta(t)$ at position $\boldsymbol{y}$. The geometric layout of the antenna elements naturally plays an important role here. Let therefore the $j^{\text {th }}$ transmit element be located at position $\boldsymbol{z}_{j}$, and the $i^{\text {th }}$ receive element be located at position $\boldsymbol{x}_{i}$. In the current analysis we use a linear scattering model often known as the distorted-wave Born approximation.[19] If we define a $(m \times n)$ matrix $G(\boldsymbol{y}, t)$ with matrix elements

$$
G_{i j}(\boldsymbol{y}, t)=\int g\left(\boldsymbol{z}_{j}, \boldsymbol{y}, \tau^{\prime}\right) \partial_{t}^{2} g\left(\boldsymbol{y}, \boldsymbol{x}_{i}, t-\tau^{\prime}\right) \mathrm{d} \tau^{\prime},
$$

then

$$
\boldsymbol{m}(t)=\mathcal{H}(V) \boldsymbol{s}(t)=\int G(\boldsymbol{y}, t-\tau) V(y) \mathrm{d} \boldsymbol{y} \boldsymbol{s}(\tau) \mathrm{d} \tau .
$$

Integration in Eq. (5) is understood to be element-wise.

\section{Target and clutter}

The above formalism allows us to utilize a physics-based model for the background which in principle can have an arbitrary level of detail. It is not reasonable, however, to expect that our background model will account for all details of the scene except for the target. As outlined in the introduction, this suggests that the reflectivity function should be divided into two parts

$$
V(\boldsymbol{x})=T(\boldsymbol{x})+C(\boldsymbol{x}),
$$

where $T(\boldsymbol{x})$ represents target and $C(\boldsymbol{x})$ represents clutter. Our definition of clutter thus includes contrubutions due to a compromise between model fidelity and tractability: deviations between the background and our model for the background. Our real interest lies in recovering $T$, while suppressing $C$.

In our development we assume that $T(\boldsymbol{x})$ and $C(\boldsymbol{x})$ are realizations of second-order random fields with known first- and second-order statistics. It should be clear that the background may be defined in such a way that the first-order statistics of the reflectivity functions are zero. Thus, without loss of generality, we will assume that the processes have zero-mean and known auto-correlation functions

$$
\begin{aligned}
& R_{T}\left(\boldsymbol{y}_{1}, \boldsymbol{y}_{2}\right)=\mathrm{E}\left[T\left(\boldsymbol{y}_{1}\right) \overline{T\left(\boldsymbol{y}_{2}\right)}\right] \\
& R_{C}\left(\boldsymbol{y}_{1}, \boldsymbol{y}_{2}\right)=\mathrm{E}\left[C\left(\boldsymbol{y}_{1}\right) \overline{C\left(\boldsymbol{y}_{2}\right)}\right] .
\end{aligned}
$$

Furthermore, we will assume that the fields $T$ and $C$ are statistically independent, i.e.,

$$
\begin{aligned}
R_{V}\left(\boldsymbol{y}_{1}, \boldsymbol{y}_{2}\right) & =\mathrm{E}\left[\left(T\left(\boldsymbol{y}_{1}\right)+C\left(\boldsymbol{y}_{1}\right)\right) \overline{\left(T\left(\boldsymbol{y}_{2}\right)+C\left(\boldsymbol{y}_{2}\right)\right)}\right] \\
& =R_{T}\left(\boldsymbol{y}_{1}, \boldsymbol{y}_{2}\right)+R_{C}\left(\boldsymbol{y}_{1}, \boldsymbol{y}_{2}\right) .
\end{aligned}
$$

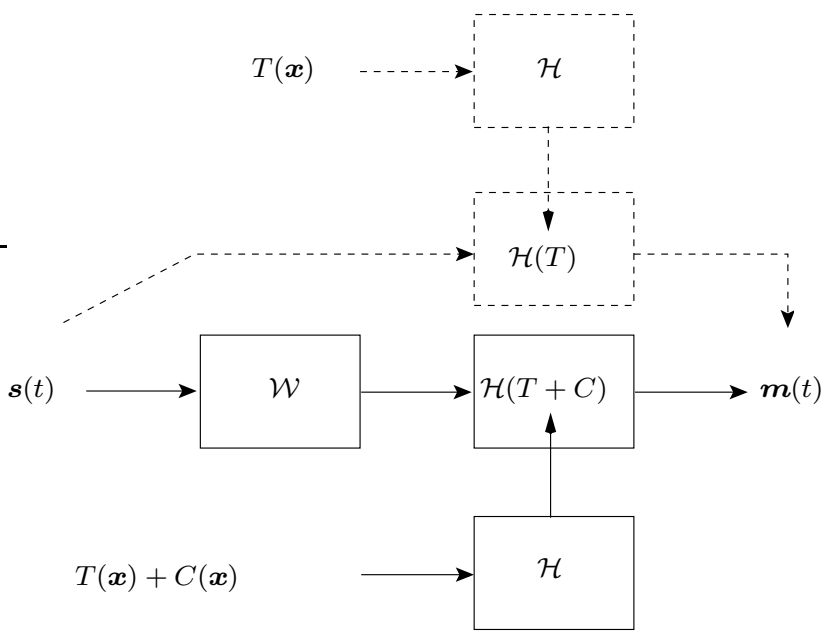

Fig. 2. Block diagram for the waveform preconditioning with the operator $\mathcal{W}$. Solid arrows indicate the signal path from waveform, through preconditioning operator and then transmitted into an environment with system response $\mathcal{H}(T+C)$. Dashed lines indicate the "ideal" signal path which is emulated by applying the preconditioning operator.

In this work we choose to reject clutter in the minimum-mean-squareerror (MMSE) sense. To be more specific: under mild assumptions about the transmit waveforms and the reflectivity functions we can show that $\mathcal{H}(V)$ is a Hilbert-Schmidt (HS) operator [20, Ch. 6.2]. Our goal is therefore to determine a linear operator $\mathcal{W}$ which minimizes $\Delta(W)$

$$
\Delta(\mathcal{W}):=\|\mathcal{H}(T+C) \mathcal{W}-\mathcal{H}(T)\|_{\mathrm{HS}}^{2} .
$$

We achieve clutter rejection by employing the transmit waveform $\mathcal{W} s$ instead of $s$. Our approach is illustrated in Fig. 2.

\section{THE PRECONDITIONING OPERATOR}

In this section we will derive an explicit expression for $\mathcal{W}$ which minimizes the error $\Delta(W)$ as defined in Eq. (11). In order for the analysis to hold, we make certain assumptions about the transmit waveforms and the scattering environment. These assumptions are mild enough to be satisfied for any practical application.

First of all, we consider transmit waveforms which have finite length, and which has sufficient spectral decay as a function of frequency. Let the temporal Fourier transfrom of $s(t)$ be denoted by $\hat{s}(\omega)$. Sufficient asymptotic decay is guaranteed if we employ the following weighted inner product on the space of transmit waveforms [21]

$$
\left\langle\boldsymbol{s}_{1}, \boldsymbol{s}_{2}\right\rangle=\int\left(1+\omega^{6}\right) \hat{\boldsymbol{s}}_{2}^{\mathrm{H}}(\omega) \hat{\boldsymbol{s}}_{1}(\omega) \mathrm{d} \omega .
$$

Here $\mathrm{H}$ denotes conjugate transpose of the vector quantities. Second, it is convenient to assume that no echoes emerge from outside a sufficiently large region, i.e., that the reflectivity function has compact support.

Under the aforementioned assumptions about the waveforms and scattering environment, we can show that $\mathcal{H}(V)$ is a HS operator [21].

Let $\left\{\boldsymbol{e}_{k}(t)\right\}$ be a basis for the space of transmit vectors. The HS norm of $\mathcal{H}(V)$ can be computed as [1]

$$
\|\mathcal{H}(V)\|_{\mathrm{HS}}^{2}=\sum_{k} \int \boldsymbol{e}_{k}^{\mathrm{H}}(t) \mathrm{E}\left[\mathcal{H}(V)^{*} \mathcal{H}(V)\right] e_{k}(t) \mathrm{d} t .
$$

Here $*$ denotes the adjoint, and $\mathrm{H}$ denotes the conjugate transpose of a vector. 
We now determine the operator $\mathcal{W}$ which satisfies Eq. (11). If we require that

$$
\Delta(\mathcal{W}+\delta \mathcal{W})-\Delta(\mathcal{W})=\mathcal{O}\left(\|\delta \mathcal{W}\|_{\mathrm{HS}}^{2}\right)
$$

for all perturbations $\delta \mathcal{W}$, we find that the optimum is

$$
\mathcal{W}=\mathrm{E}\left[\mathcal{H}(T+C)^{*} \mathcal{H}(T+C)\right]^{-1} \mathrm{E}\left[\mathcal{H}(T)^{*} \mathcal{H}(T)\right] .
$$

This operator may be applied to any transmit vector to yield a new transmit vector; $\mathcal{W}$ is a bounded linear operator on the space of transmit vectors.

\section{TIME-REVERSAL PRECONDITIONING}

In [21], we further evaluated $\mathcal{W}$ from Eq. (15) in terms of the correlation functions for target and clutter as defined in Eqns. (7) and (8). This lead to an explicit expression for the preconditioning operator. However, it required us to obtain information about the second-order statistics of target and clutter, as well as the Green's function for the background medium. Here we will outline a different approach which allows us to estimate the preconditioning operator from scattering measurements directly.

First, from Eq. (5) we see that $\mathcal{H}(T+C)=\mathcal{H}(T)+\mathcal{H}(C)$. We use this fact to write

$$
\mathcal{H}(T)=\mathcal{H}(T+C)-\mathcal{H}(C) .
$$

Inserting Eq. (16) into Eq.(15) we arrive at

$$
\mathcal{W}=I-\mathrm{E}\left[\mathcal{H}(T+C)^{*} \mathcal{H}(T+C)\right]^{-1} \mathrm{E}\left[\mathcal{H}(C)^{*} \mathcal{H}(C)\right] .
$$

The significant difference between Eq. (15) and (17) is that in Eq. (17) we have replaced $\mathrm{E}\left[\mathcal{H}(T)^{*} \mathcal{H}(T)\right]$ by $\mathrm{E}\left[\mathcal{H}(C)^{*} \mathcal{H}(C)\right]$, This is important, as $\mathcal{H}(T)$ corresponds to scattering from a target without the presense of clutter, and is infeasible to measure. On the other hand, $\mathcal{H}(C)$ corresponds to scattering from clutter. This can be observed at times when there is no target present.

By definition $\boldsymbol{m}(t)=\mathcal{H}(C) \boldsymbol{s}(t)$. Furthermore, if we interchange the roles of transmitters and receivers, and transmit $\boldsymbol{m}(-t)$, this corresponds to employing the transpose of the matrix kernel $G$ in Eq. (5), i.e.,

$$
\boldsymbol{q}(t)=\int G^{\mathrm{T}}(\boldsymbol{y}, t-\tau) C(y) \mathrm{d} \boldsymbol{y} \boldsymbol{m}(-\tau) \mathrm{d} \tau .
$$

Observe also that if we take the inner product between $\mathcal{H}(C) s(t)$ and an element $\boldsymbol{f}(t)$ in $\mathrm{L}^{2}(\mathbb{R})$, then by the Plancherel's identity

$$
\langle\boldsymbol{f}, \mathcal{H}(C) \boldsymbol{s}\rangle=\int \hat{\boldsymbol{f}}^{\mathrm{H}}(\omega) \hat{G}(\boldsymbol{y}, \omega) C(\boldsymbol{y}) \mathrm{d} \boldsymbol{y} \hat{\boldsymbol{s}}(\omega) \mathrm{d} \omega .
$$

Here $^{\wedge}$ is used to indicate temporal Fourier transform. If we now reorganize the matrix-vector multiplication

$$
\begin{aligned}
\langle\boldsymbol{f}, \mathcal{H}(C) \boldsymbol{s}\rangle & =\int\left(\hat{G}^{\mathrm{H}}(\boldsymbol{y}, \omega) \hat{\boldsymbol{f}}(\omega)\right)^{\mathrm{H}} C(\boldsymbol{y}) \mathrm{d} \boldsymbol{y} \hat{\boldsymbol{s}}(\omega) \mathrm{d} \omega \\
& =\int\left(\overline{\hat{G}^{\mathrm{T}}(\boldsymbol{y}, \omega) \overline{\hat{\boldsymbol{f}}(\omega)}}\right)^{\mathrm{H}} C(\boldsymbol{y}) \mathrm{d} \boldsymbol{y} \hat{\boldsymbol{s}}(\omega) \mathrm{d} \omega .
\end{aligned}
$$

Now we insert the expression for the inner product on the space of transmit waveforms from Eq. (12), and see that

$$
\langle\boldsymbol{f}, \mathcal{H}(C) s\rangle=\left\langle\mathcal{H}(C)^{*} \boldsymbol{f}, \boldsymbol{s}\right\rangle_{\mathrm{S}},
$$

where the temporal Fourier transform of the adjoint $\mathcal{H}(C)^{*}$ applied to $f$ is

$$
\int \frac{1}{1+\omega^{6}}\left(\overline{\hat{G}^{\mathrm{T}}(\boldsymbol{y}, \omega) \overline{\hat{\boldsymbol{f}}(\omega)}}\right) C(\boldsymbol{y}) \mathrm{d} \boldsymbol{y}
$$

We should now point out that complex conjugation in the temporal frequency domain corresponds to time-reversal in the time-domain.
Hence, by comparing Eqns. (18) and (23), we conclude that $\mathcal{H}(C)^{*}$ applied to $f$ is computed by re-transmitting a time-reversed version of $f$, and then time-reversing the corresponding measured scattering. In order to end up in the right space, we apply a zero-phase filter with freqency responce $1 /\left(1+\omega^{6}\right)$.

Under the assumption that there is no target present in the scene, the following algorithm will allow us to obtain $\mathcal{H}(C)^{*} \mathcal{H}(C)$ applied to a transmit vector $s$ :

1) Transmit waveform $\boldsymbol{s}(t)$ and obtain measurement $\boldsymbol{m}(t)$.

2) Interchange the role of transmitters and receivers

3) Transmit waveform $\boldsymbol{m}(-t)$ and obtain measurement $\boldsymbol{p}(t)$.

4) Get $q(t)=\mathcal{H}(C)^{*} \mathcal{H}(C) s$ by applying the zero-phase filter with temporal frequency response $1 /\left(1+\omega^{6}\right)$ to $p(-t)$.

Averaging over repeated evaluations will produce the expected value $\mathrm{E}\left[\mathcal{H}(C)^{*} \mathcal{H}(C)\right] s$. Iterative application of this algorithm allows us to determine the eigenvalues/eigenvectors $\left\{\left(\lambda_{k}, \boldsymbol{u}_{k}\right)\right\}$ of $\mathrm{E}\left[\mathcal{H}(C)^{*} \mathcal{H}(C)\right]$, e.g., by a power-method.

The same procedure will allow us to determine the eigenvalues/eigenvectors $\left\{\left(\sigma_{k}, \boldsymbol{v}_{k}\right)\right\}$ of $\mathrm{E}\left[\mathcal{H}(T+C)^{*} \mathcal{H}(T+C)\right]$ if a target is present in the scene. Finally, the preconditioning operator in Eq. (17) applied to $s$ is found as

$$
\mathcal{W} \boldsymbol{s}=\boldsymbol{s}-\sum_{l} \sum_{k} \frac{\lambda_{k}}{\sigma_{l}}\left\langle\boldsymbol{s}, \boldsymbol{u}_{k}\right\rangle\left\langle\boldsymbol{u}_{k}, \boldsymbol{v}_{l}\right\rangle \boldsymbol{v}_{l} .
$$

Loosely speaking, $\mathcal{W}$ emphasizes the parts of $s$ residing the subspaces in which the signal-to-clutter ratio is high [1].

\section{NUMERICAL SIMULATION}

In order to demonstrate the clutter-suppression obtained with our waveform preconditioning operator, we have performed a set of numerical simulations. In these simulations we want to recover the target $T$ from scattering measurements made with two transmitters and ten receivers. The transmitters and receivers were placed equally spaced on an arc around the target. This simulation setup is illustrated in Fig. 1.

From the two transmitters we transmitted short chirp signals: transmitter 1 emitted a linear up-chirp, while transmitter 2 emanated a linear down-chirp. All dimensions of the experiment were normalized according to a unit length scale $\lambda$.

As a target we chose a square with sides $1.5 \lambda \times 1.5 \lambda$. From this target model we constructed a target spectrum as if the target were a realization of a stationary random field. A high-frequency version of the stationary stochastic target model was then constructed and used to simulate different realizations of the surrounding clutter. This construction is explained further in Yazici et al. [22]. The compact support of the clutter was imposed by applying a spatial mask. For our purpose we used a region of $5 \lambda \times 5 \lambda$ around the target. Finally, the radius of the arc on which the antenna elements were placed was set to $10 \lambda$.

The preconditioning operator was constructed according to Eq. (24) from 50 estimated eigenvalues and eigenvectors for $\mathrm{E}\left[\mathcal{H}(C)^{*} \mathcal{H}(C)\right]$ and $\mathrm{E}\left[\mathcal{H}(T+C)^{*} \mathcal{H}(T+C)\right]$ by a Monte-Carlo approach. The spatial discretization for each scattering simulation was 15 samples per wavelength $\lambda$.

The signal-to-clutter ratio (SCR) in our simulations was set to $-6 \mathrm{~dB}$, when defined according to

$$
\mathrm{SCR}=10 \log \left(\frac{\int \mathrm{E}\left[|T(\boldsymbol{x})|^{2}\right] \mathrm{d} \boldsymbol{x}}{\int \mathrm{E}\left[|C(\boldsymbol{x})|^{2}\right] \mathrm{d} \boldsymbol{x}}\right) .
$$

The performance of the preconditioning was then evaluated by observing the square error in the reconstructed image when compared to the true reflectivity function. The mean-square-error (MSE) was 

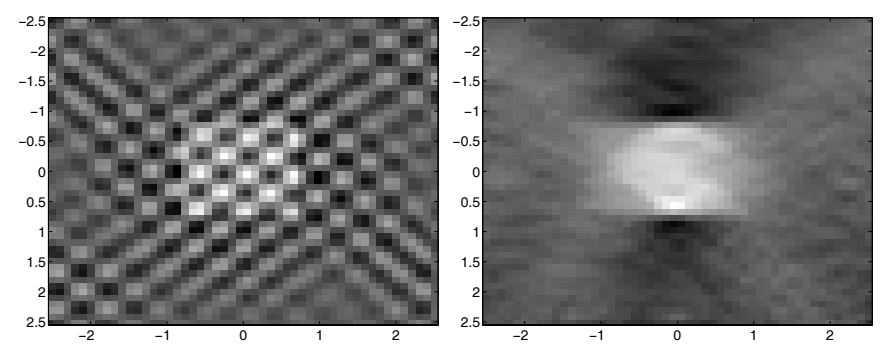

Fig. 3. Target with clutter (left) and reconstruction of target from clutter-free scattering (right)
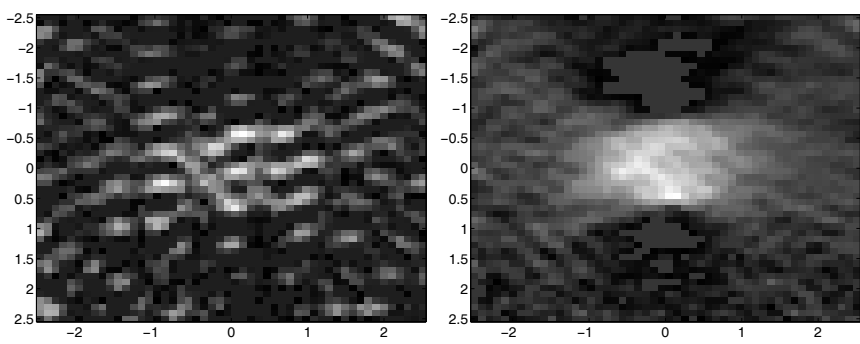

Fig. 4. Reconstruction results for a single realization of clutter. Left: image from scattering with original chirp waveform. Right: image from scattering with preconditioned chirp waveform

estimated by averaging over 10 clutter realizations. We computed the MSE according to

$\operatorname{MSE}(\mathcal{W})=10 \log \left(\frac{\int \mathrm{E}\left[\left|\left[\mathcal{H}^{-1} \mathcal{H}(T+C) \mathcal{W}\right](\boldsymbol{x})-T(\boldsymbol{x})\right|^{2}\right] \mathrm{d} \boldsymbol{x}}{\int \mathrm{E}\left[|T(\boldsymbol{x})|^{2}\right] \mathrm{d} \boldsymbol{x}}\right)$

We used an imaging reconstruction algorithm which is based on minimising the MSE of the final image.[21] The images were reconstructed on a grid with 10 samples per wavelength.

Figure 3 shows the target embedded in clutter, as well as a reconstructed image based on scattering without clutter. The MSE is in this case was $3.5 \mathrm{~dB}$.

Figure 4 show reconstruction results for scattering with clutter. Preconditioning of the transmit waveform improves image quality from $8.9 \mathrm{~dB}$ to $4.3 \mathrm{~dB}$ when measured using the MSE defined in Eq. (25).

Figure 5 shows the spectrum of one of the transmit waveform that were employed in this experiment.

\section{DISCUSSION AND CONCLUDING REMARKS}

In this work we have separated the reflectivity function into two distinct classes: target and clutter. The clutter essentially produces unwanted scattering which in turn degrades the final result of the reconstructed image. If scattering from clutter can be removed from the measurement, the end result will be improved. Our preconditioning operator can be applied to any set of transmit vectors in order to optimally reject scattering from clutter in the MMSE sense.

In previous implementations we have employed information about the scene in the form of the Green's function. This Green's function was used to map second-order statistics of the clutter and target to the space of transmit vectors and thereby construct the preconditioning operator. Here we avoid explicitly using the environment model by instead evaluating the operators by a time-reversal approach.

We address the problem of optimally modifying the transmit waveform when the transmit power is limited. Obviously, after preconditioning the resulting waveform may not have the same

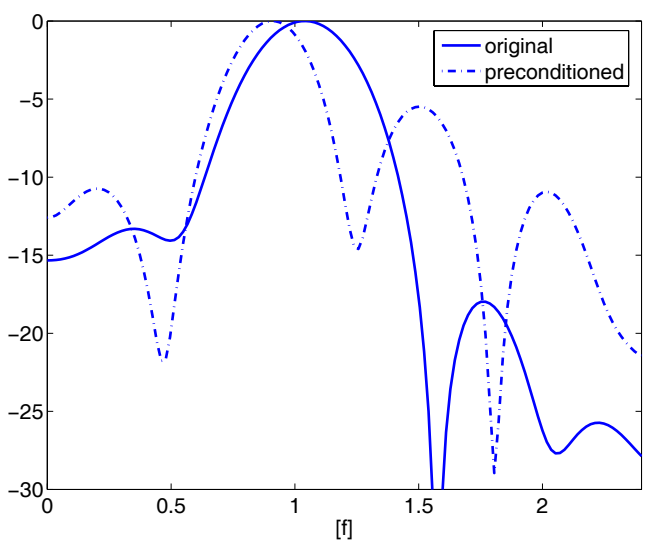

Fig. 5. Spectrum magnitude (right) for the original waveform, and for the preconditioned waveform.

strength. However, this is trivially amended by proper normalization of the preconditioned waveform. As a result, the transmit vector will contain the same amount of power, but will minimize scattering from clutter.

When we perform clutter rejection we identify a transmit-vector subspace where the signal-to-clutter (SCR) ratio is high. The fact that the signals which are employed at each transmitter are transmitted simultaneously, and that they are not orthogonal imply that there is a great deal of ambiguity in the data with respect to the correct time-offlight for a given echo. We therefore have limited ability to determine the correct source-reciever pair for a given echo. As we are forming the images using a our limited-angle tomographic approach, we do not rely on the ability to resolve the source-reciever ambiguity.[21]

An important feature of the preconditioning approach is the improved SCR of the scattering. Hence, for a fixed total transmit power, the SCR may be improved in the final image. Alternatively, for a given signal-to-noise ratio in the final image the total transmit power can be reduced. This is of interest in applications where it is desirable to keep the transmit power as low as possible, e.g., to reduce transmitter vulnerability/detectability.

The underlying propagation model which we have used for this work is derived from a scalar wave equation. This is a commonlyused model for many radar applications where polarization effects may be ignored. In order to get explicit expressions in terms of Green's functions, a linearized scattering model was used, namely the distorted-wave Born approximation (DWB). Note, however, that the operator norm which we used to determined the preconditioning operator will make sense also without the DWB. The time-reversal principle also holds in other pulse-echo applications. Our work therefore has applications also in other areas such as ultrasound, sonar and microwave imaging.

\section{ACKNOWLEDGMENTS}

We are grateful to Air Force Office of Scientific Research ${ }^{1}$ (AFOSR) and the Defense Advanced Research Projects Agency (DARPA) for supporting this work under the agreements FA955004-1-0223, FA9550-06-1-0017 and FA8750-05-2-0285.

${ }^{1}$ Consequently, the US Government is authorized to reproduce and distribute reprints for governmental purposes notwithstanding any copyright notation thereon. The views and conclusions contained herein are those of the authors and should not be interpreted as necessarily representing the official policies or endorsements, either expressed or implied, of the Air Force Research Laboratory or the US Government. 


\section{REFERENCES}

[1] T Varslot, B Yazıcı, C E Yarman, M Cheney, and L Scharf, "Waveform preconditioning for clutter rejection in multipath for sparse distributed apertures," in Proc. SPIE Defence and Security Conference 2007, 2007.

[2] B Yazıcı and G Xie, "Wideband extended range-Doppler imaging and waveform design in the presence of clutter and noise," IEEE Trans. Inf. Theory, vol. 52, no. 10, 2006.

[3] M Fink, "Time-reversed acoustics," Scientific American, vol. 281, pp. 91-97, Nov 1999.

[4] M Fink, "Time reversal of ultrasonic fields - part I: Basic prinsiples," IEEE Trans. Ultrason. Ferroelectr. Freq. Control, vol. 39, pp. 555-567, 1992.

[5] C Prada, J L Thomas, and M Fink, "The iterative time reversal process: analysis of convergence," J. Acoust. Soc. Am., vol. 97, no. 1, pp. 62-71, January 1995.

[6] M Tanter, J-L Thomas, and M Fink, "Time reversal and the inverse filter," J. Acoust. Soc. Am., vol. 108, no. 1, pp. 223-234, July 2000.

[7] G Montaldo, D Palacio, M Tanter, and M Fink, "Building threedimensional images using a time-reversal chaotic cavity," IEEE Trans. Ultrason. Ferroelectr. Freq. Control, vol. 52, no. 9, pp. 1489-1497, September 2005.

[8] G F Delmann, H C Song, S Kim, W S Hodgkiss, W A Kuperman, and T Akal, "Underwater acoustic communications using time reversal," IEEE J Oceanic Engineering, vol. 30, no. 4, pp. 852-864, 2005.

[9] H C Song, W S Hodgkiss, W A Kuperman, M Stevenson, and T Akal, "Improvement of time-reversal communications using adaptive channel equalizers," IEEE J Oceanic Eng., vol. 31, no. 2, pp. 487-496, 2006.

[10] D Liu, G Li, Y Chen, S Vasudevan, W Jones, Q Liu, J Krollik, and L Carin, "Electromagnetic time-reversal imaging of a target in a cluttered environment," IEEE Trans. Ant. Prop., vol. 53, no. 9, pp. 3058-3066, 2005.

[11] A Devaney, "Time reversal imaging of obscured targets from multistatic data," IEEE Trans. Ant. Prop., vol. 53, no. 5, pp. 1600-1610, 2005.

[12] P Kosmas and C M Rappaport, "A matched-filter fdtd-based time reversal approach for microwave breast cancer detection," IEEE Trans. Ant. and Prop., vol. 54, no. 4, pp. 1257-1264, 2006.

[13] P Blomgren, G Papanicolaou, and H Zhao, "Super-resolution in timereversal acoustics," J. Acoust. Soc. Am., vol. 111, no. 1, pp. 203-248, 2002.

[14] C Oestges, A D Kim, G Papanicolaou, and A.J. Paulraj, "Characterization of space-time focusing in time reversed random fields," IEEE Trans. Ant. and Prop., vol. 53, no. 1, pp. 283-293, 2005.

[15] G Papanicolaou, L Ryzhik, and K Solna, "Statistical stability in time reversal," SIAM J, Appl. Math., vol. 64, pp. 1133-1155, 2004.

[16] G Forney and M Eyuboglu, "Combined equalization and coding using precoding," IEEE Comm Mag, Dec 1991.

[17] H Harashima and H Miyakawa, "Matched transmission technique for channels with intersymbol interference," IEEE Trans. Commun., vol. COM-20, pp. 774-780, 1972.

[18] M Tomlinson, "New automatic equalizer employing modulo arithmetic," Electron. Lett., vol. 7, pp. 138-139, 1971.

[19] J H Taylor, Scattering Theory, Wiley, New York, 1972.

[20] J Weidmann, Linear Operators in Hilbert Spaces, Springer-Verlag, New York, 1980.

[21] T Varslot, B Yazıcı, and M Cheney, "Pulse-echo imaging with distributed apertures in multi-path environments," (preprint), 2006.

[22] B Yazıc1, M Cheney, and C E Yarman, "Synthetic aperture inversion for an arbitrary flight trajectory in the presence of noise and clutter," Inverse Problems, vol. 22, no. 5, pp. 1705-1729, 2006. 